\title{
A case of Churg-Strauss syndrome: tissue diagnosis established by sigmoidoscopic rectal biopsy
}

\author{
E J Leen, P J Rees, J D Sanderson, M L Wilkinson, M I Filipe
}

\begin{abstract}
A case is presented of Churg-Strauss syndrome in a young man in whom the definitive diagnostic procedure was a full thickness sigmoidoscopic rectal biopsy, with submucosal sampling. Gastrointestinal changes in Churg-Strauss syndrome, a rare systemic illness characterised by asthma, blood and tissue eosinophilia, vasculitis, and granulomatous inflammation are common but poorly reported. The endoscopic and histopathological features of a case are described and emphasise the potential value of a limited sigmoidoscopy in establishing the diagnosis, when lower gastrointestinal symptoms are present.

(Gut 1996; 38: 299-301)
\end{abstract}

Keywords: Churg-Strauss syndrome, vasculitis, endoscopy, rectal biopsy.

\section{Case report}

A 23 year old white man presented with a one week history of bloody diarrhoea, anorexia, and lower abdominal pain. Increasing breathlessness and a cough productive of yellow bloody sputum were also noted. The patient had been well until 19 months previously when asthma was diagnosed on clinical grounds. There was evidence of allergy to cats and wine but no history of hay fever. The asthma was not well controlled with inhaled bronchodilators and corticosteroids and required regular courses of oral prednisolone therapy in doses of between 10 and $30 \mathrm{mg}$ daily.

Ten months prior to the current episode, he required urgent admission because of increasing wheeze, dyspnoea, and haemoptysis. He also developed lower abdominal pain and rectal bleeding. On examination, tachycardia, tachypnoea, inspiratory crackles, and expiratory wheezes were present. Investigations showed hypoxia $\left(\mathrm{PO}_{2}\right.$ of $6.3 \mathrm{kPa}$ on room air), bilateral nodular lower zone shadows on chest $x$ ray, a leucocytosis of $28.7 \times 10^{9} / 1$ with an eosinophil count of $5.0 \times 10^{9} / 1$, a haemoglobin concentration of $12.4 \mathrm{~g} / \mathrm{dl}$, and an erythrocyte sedimentation rate of $18 \mathrm{~mm}$ in the first hour. A temperature of $38.6^{\circ} \mathrm{C}$ was noted on admission and he was given oxygen, intravenous corticosteroids and antibiotics, and oral prednisolone. He made a good recovery and was discharged one week later with a normal chest $x$ ray, eosinophil count, and blood gases. Transbronchial biopsy specimens, which were taken on the third day after admission, showed non-specific changes only with no vasculitis. Serological tests for aspergillus were negative, the patient had never been to the tropics and was taking no drugs associated with eosinophilia. His HIV status was not known but he had no known risk factors for this infection.

In the three months after this episode he developed persistent lower leg muscle pain with left leg swelling and a persistent right knee effusion. Serological tests for rheumatoid factor, antinuclear factor and antineutrophil cytoplasmic antibody were negative. Knee and sacroiliac joint radiographs were normal.

$\mathrm{He}$ was seen at the gastroenterology clinic two months after his admission because of some continuing lower abdominal pain and diarrhoea. His symptoms were quite mild and rectal biopsy showed only non-specific glandular distortion. This biopsy specimen consisted of mucosal tissue only without submucosa. Thus, although a systemic vasculitic episode was suspected as the cause of the clinical story, a definite diagnosis could not be established. The patient was maintained with oral corticosteroids with a persistent pattern of worsening of his respiratory symptoms once the daily prednisolone dose was reduced to around $10 \mathrm{mg}$.

The 'diagnostic' episode that forms the basis of this report was preceded by a one week history of increasing dyspnoea, bloody diarrhoea, and abdominal pain. He became increasingly dyspnoeic and a chest $x$ ray showed an alveolar filling pattern in the left mid and lower zones as well as right midzone shadows. Eosinophil count was normal but he had increased his prednisolone dose five days earlier. A rigid sigmoidoscopy to $14 \mathrm{~cm}$ showed severe proctitis with contact haemorrhage. A $4 \mathrm{~mm}$ rectal biopsy specimen was taken, fixed in $10 \%$ formalin, routinely processed, and stained with haematoxylin and eosin, elastic van Gieson, Ziehl Nielsen, and Martius Scarlet Blue.

\section{Histopathology}

(Figures 1-5) The mucosal zone is largely normal apart from focal glandular loss and small lamina propria aggregates of eosinophils and histiocytes. The submucosa shows the diagnostic histological features with broad areas of coagulative necrosis surrounded by a rim of palisaded histiocytes and epithelioid cells (necrotising granulomas), a heavy infiltrate of eosinophils, small thrombi, and disrupted vessels with inflammation in vascular walls (vasculitis). A Ziehl Nielsen stain for
Correspondence to: Dr E Leen, Histopathology Guy's House, Guy's Hospital, London SE1 9RT. Accepted for publication 24 July 1995 


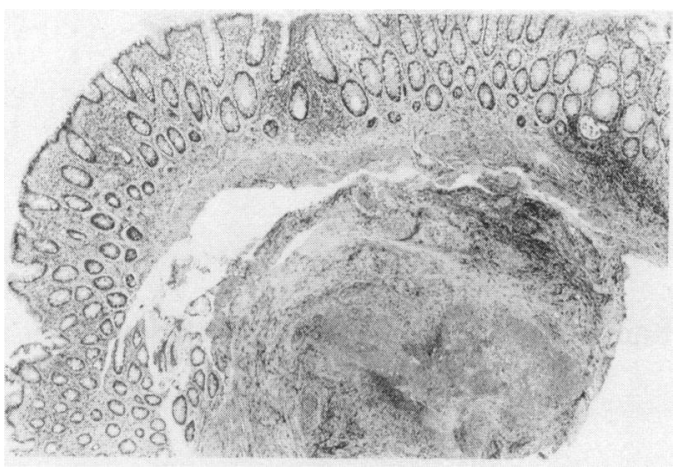

Figure 1: $(\times 100)$ Photomicrograph with a largely normal mucosa (above) overlying an expanded submucosa: note the intense inflammatory infiltrate just beneath the muscularis mucosa (middle right).

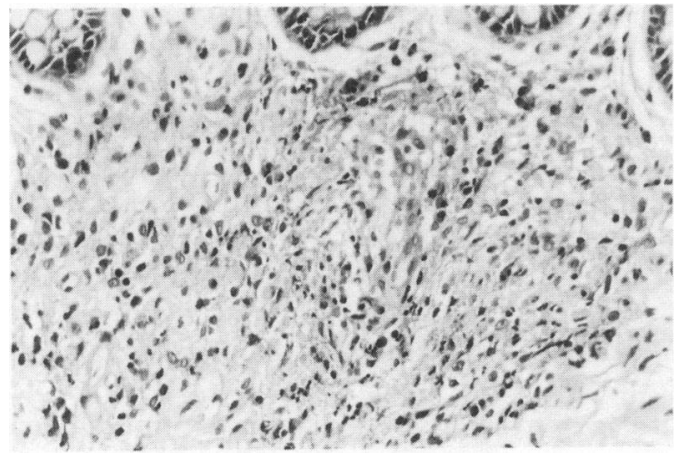

Figure 2: $(\times 400) A$ high power view of the deep mucosa in which a crypt has been largely destroyed by an infiltrate of eosinophils and histiocytes.

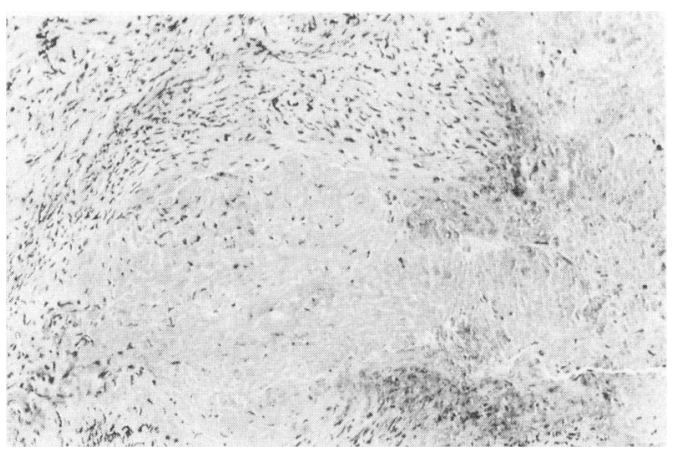

Figure 3: $(\times 250)$ A focus of 'geographic' coagulative necrosis in the submucosa surrounded by a palisaded arrangement of histiocytes.

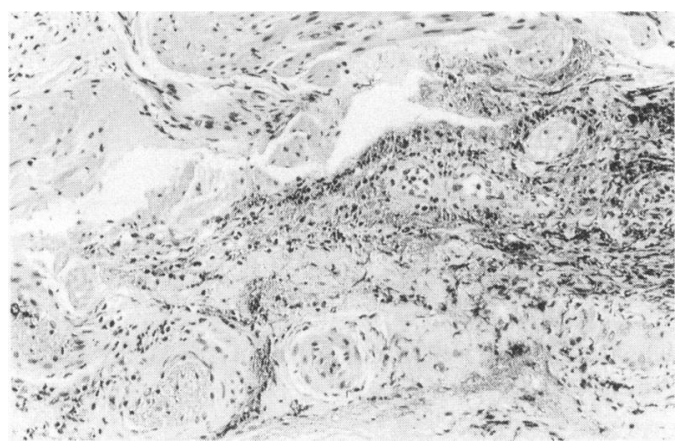

Figure 4: $(\times 250) A$ view of the inflamed area referred to in Fig 1 showing, beneath the muscularis mucosae (top) submucosal infiltration by eosinophils with thrombi in small vessels. There is pronounced eosinophilia of connective tissue (bottom right) due to eosinophil degranulation.

mycobacteria was negative. The histological features, taken in conjunction with the clinical picture were diagnostic of Churg-Strauss vasculitis. The patient's prednisolone dose was increased to $60 \mathrm{mg}$ daily (from $10 \mathrm{mg}$ ). His respiratory and bowel symptoms settled on this regimen. A full colonoscopy, performed 12

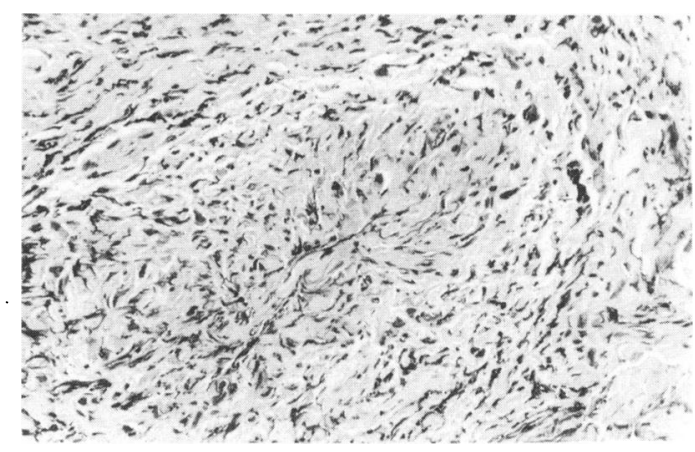

Figure 5: (×400) A small submucosal vessel with considerable luminal narrowing, and intense inflammation in its wall.

days after the original limited sigmoidoscopy, and one week after the corticosteroid dose was increased showed a normal terminal ileum and patchy mucosal erythema from the rectum to the proximal ascending colon and caecum. In the rectum the mucosa was granular and congested with multiple small ulcers (Figs 6 and 7). Histologically, scattered eosinophil crypt abscesses were present in the sigmoid colon and mucosal vascular granulomas were noted in the rectum. The proximal colon and terminal ileum were histologically normal.

\section{Discussion}

Churg-Strauss syndrome is a disorder characterised by blood hypereosinophilia and systemic vasculitis occurring in subjects with asthma and allergic rhinitis, originally described in 1951. ${ }^{1}$ The condition is considered to be rare, but it has been pointed out that this is due to an over rigid adherence to the three major criteria described in the original series that is, (a) necrotising vasculitis, (b) tissue infiltration by eosinophils, and (c) extravascular granulomas. Lanham ${ }^{2}$ pointed out that the three histological components of the syndrome often do not coexist spatially or temporally and are only found together in life in a few cases. In a recent review of arteritis, ${ }^{3}$ Churg-Strauss syndrome is classified as a noninfectious arteritis involving small and medium sized vessels. Wegener's granulomatosis and classical polyarteritis are also in this group and may be difficult to separate from ChurgStrauss syndrome on purely histological grounds, however neither shows the blood eosinophilia or asthmatic tendency so typical of Churg-Strauss syndrome. Conversely both are more likely to develop serious renal impairment. Churg-Strauss syndrome is immunologically mediated with types I, III, and IV hypersensitivity being involved. ${ }^{3}$

The gastrointestinal manifestations of Churg-Strauss syndrome are diverse and include features of eosinophilic gastroenteritis and vasculitis. Abdominal pain, diarrhoea, and bleeding occur in 59,33 , and $18 \%$ of patients respectively. ${ }^{2}$ Histological descriptions of enteric disease are much rarer. ${ }^{4}$ Eosinophilic gastroenteritis may be a prodrome of ChurgStrauss syndrome and some reported cases of this are clinically very suggestive of 


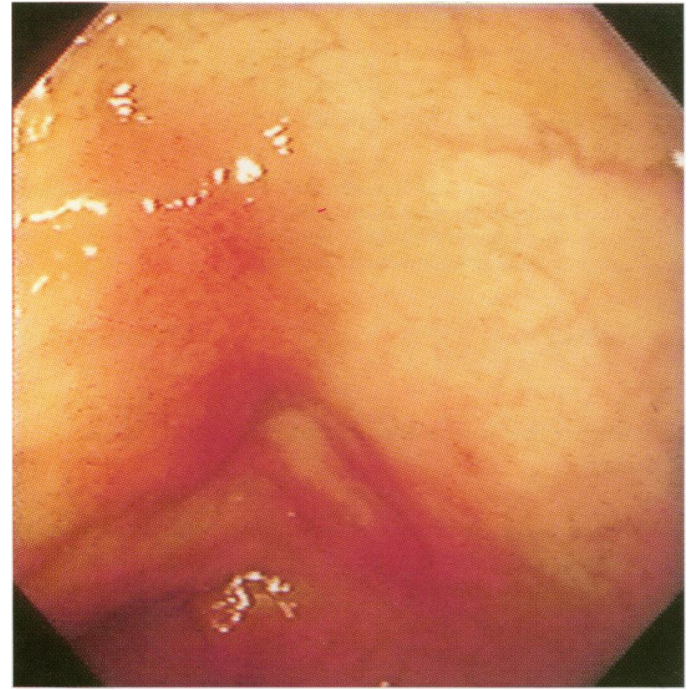

Figure 6: Endoscopic view of the sigmoid colon showing patchy mucosal erythema with loss of vascular pattern (Olympus CF200 L).

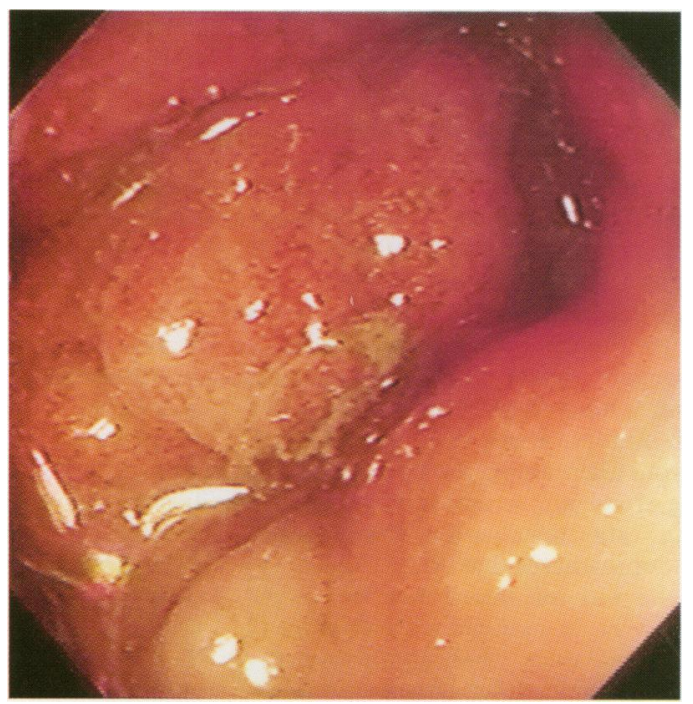

Figure 7: Endoscopic view of rectum showing an intense proctitis with congestion, erythema, and discrete mucosal ulceration (Olympus CF200 L).

Churg-Strauss syndrome. ${ }^{6}$ In eosinophilic colitis $^{7}$ usually the whole thickness of the bowel is heavily infiltrated by eosinophils but if the mucosa is spared colonoscopic diagnosis may not be possible. Eosinophilic colitis is generally a right sided disease with sparing of the rectosigmoid area. The endoscopic features of eosinophilic colitis are characterised by mucosal erythema, nodularity, and haemorrhage. ${ }^{6}$ Thus while the endoscopic appearances in this case were compatible, the considerable rectosigmoid involvement was atypical for a pure eosinophilic colitis. The best reported case of enteric Churg-Strauss syndrome ${ }^{4}$ shares many of the features of this case including, in the rectum, (a) ulceration, (b) a moderate increase in lamina propria eosinophils and plasma cells, (c) an extravascular mucosal granuloma, and (d) submucosal necrotising vascular granulomas. Features b-d described above were seen histologically in this case, and ulcers were seen on sigmoidoscopy. The patient described by Modigliani and colleagues had an exploratory laparotomy and multiple biopsies showed extensive peritoneal and omental disease. Churg-Strauss syndrome enteric disease may rarely pursue an aggressive course as shown by a recent report of small bowel necrosis requiring repeated surgical resections. $^{5}$

In conclusion, this case shows that abdominal symptoms in patients with established or suspected Churg-Strauss syndrome should be carefully evaluated and endoscopy and biopsy performed before they are ascribed to irritable bowel syndrome or the effects of the antibiotics, which are often given (as in this case) for severe respiratory exacerbations. This is of importance as severe mesenteric vasculitis is a medical emergency requiring treatment with cyclophosphamide. A tissue diagnosis is highly desirable before instituting such toxic therapy. Also lower gastrointestinal endoscopy and biopsy may be useful in establishing the diagnosis of Churg-Strauss syndrome especially when, as in this case, transbronchial biopsy is inconclusive. Limited sigmoidoscopy may be enough as the rectum seems to be preferentially involved. Submucosal sampling seems to be of crucial importance as the mucosal changes alone may be non-specific. ${ }^{49}$ Thus the presence or absence of submucosal tissue should be specifically mentioned by the histopathologist so that the clinician may know whether a truly diagnostically valid and useful specimen has been obtained. Laparoscopic biopsy may also be of value as shown by the presence of significant peritoneal and omental disease in previous reports.

1 Churg J, Strauss L. Allergic granulomatosis, allergic angiitis and periarteritis nodosa. Am 7 Pathol 1951; 27: 277-301.

2 Lanham JC, Elkon KB, Pusey CD, Hughes GR. Systemic vasculitis with asthma and eosinophilia: a clinical approach to the Churg-Strauss syndrome. Medicine 1984; 63: $65-81$.

3 Parums DV. The arteritides. Histopathology 1994; 25: 1-20.

4 Modigliani R, Muschart J-M, Galian A, Clauvel J-P, PielDesruisseaux J-L. Allergic granulomatous vasculitis (Churg-Strauss syndrome): report of a case with widespread digestive involvement. Dig Dis Sci 1981; 26: 264-70.

5 Schoretsanitis GN, Wakely DM, Maddox T, Wastell C. A case of Churg-Strauss vasculitis complicated by small bowel necrosis. Postgrad Med f 1993; 69: 828-31.

6 Partyka EK, Sanowski RA, Kozarek RA. Colonoscopic features of eosinophilic gastroenteritis. Dis Colon Rectum 1980; 23: 353-6.

7 Naylor AR, Pollet JE. Eosinophilic colitis. Dis Colon Rectum 1985; 28: 615-8.

8 Tedesco FJ, Huckaby CB, Hamby-Allen M, Ewing GC. Eosinophilic ileocolitis: expanding spectrum of eosinophilic gastroenteritis. Dig Dis Sci 1981; 26: 943-8.

9 Shimamoto C, Hirata I, Ohshiba S, Fujiwara S, Nishio M. Churg-Strauss syndrome (Allergic granulomatous angiitis) with peculiar multiple colonic ulcers. Am $\mathcal{f}$ Gastroenterol 1990; 85: 316-9. 